\title{
Schultz Polynomial, Modified Schultz Polynomial and Indices of Molecular Graph of Anthracene Based On Domination
}

\author{
G.Jayalalitha ${ }^{1}$, M.Raji ${ }^{2}$ \\ ${ }^{1}$ Professor, Department of Mathematics, VELS Institute of Science, Technology \& Advanced Studies, \\ Chennai, India. \\ Email: ragaji94@yahoo.com \\ ${ }^{2}$ Research Scholar, Department of Mathematics, VELS Institute of Science, Technology \& Advanced Studies, \\ Chennai, India. \\ Email: rajialagumurugan@gmail.com
}

\begin{abstract}
Consider a Graph $G$ with vertex set $V=V(G)$ and edge set $E=E(G)$.Here in the Molecular Graph, vertices represent atoms and edges represent bonds. In this paper, Schultz Polynomial, Modified Schultz Polynomial of the molecular graph of Anthracene are obtained by using Minimum Dominating Distance Matrix and also their Indices is calculated.
\end{abstract}

Keywords: Dominating set, Minimum Dominating Distance matrix, Schultz Polynomial, Modified Schultz Polynomial, Schultz Index, Modified Schultz Index.

\section{INTRODUCTION}

Graph theory can be used to model many relations in real time systems. A chemical graph is a mathematical structure which provides a pictorial representation of a molecule, taking into account the internal connectivity of atoms in the molecule through bonds (Damir vukicevic, Nenad Trinajstic, 2004).Topological indices are real numbers related to a structural graph of a molecule. Such indices based on the distances in graph are widely used for establishing relationships between the structure of a molecular graph and their physicochemical properties. Topological indices are used in biology and chemistry between physicochemical properties of organic compounds and the index of their molecular graphs. Benzenoid graphs are graphs pertaining to the network constructed by arranging congruent regular hexagons in a plane, so that two hexagons are either disjoint or have one edge in common(S. Klavzar, I. Gutman,1996). Benzenoid Graph is formed by the vertices in edges lying on $\mathrm{C}$ and in the interior of C.A Linear Benzenoid Chain is a set of hexagons arranged on a horizontal line where each pair of

$$
[\mathrm{A}]_{\mathrm{ij}}= \begin{cases}1 & \text { if } \mathrm{i} \neq \mathrm{j} \text { and } \mathrm{e}_{\mathrm{ij}} \in \mathrm{E}(\mathrm{G}) \\ 0 & \text { if } \mathrm{i}=\mathrm{j} \text { and } \mathrm{e}_{\mathrm{ij}} \in \mathrm{E}(\mathrm{G})\end{cases}
$$

adjacent hexagons share a vertical edge (NisreenBukhary,2010).

\section{PRELIMINARIES}

2.1 Graph (Arumugam.S 2014):

A graph $\mathrm{G}=(\mathrm{V}, \mathrm{E})$ consists of a nonempty set $\mathrm{V}$ of vertices and a set $\mathrm{E}$ of edges.

\subsection{Dominating Set}

A set $D$ in a graph ' $G$ ' is a dominating set if each vertex is either in $\mathrm{D}$ or adjacent to a vertex in D.

\subsection{Minimum Dominating Set}

Any dominating set with minimum cardinality is called a minimum dominating set.

2.4 Adjacency Matrix (Qian-Nan Hu, Yi-Zeng Liang and Kai-Tai Fang, 2003)

The adjacency matrix of a molecular graph having $\mathrm{N}$ vertices is a square $\mathrm{N} x \mathrm{~N}$ symmetric matrix which is defined as

Where $e_{i j}$ is the edge between $i$ and $j$ and $E(G)$ is the set of edges of the graph $G$.

\subsection{Distance Matrix}


The Distance Matrix (D) of a Molecular graph having $\mathrm{N}$ vertices is a real symmetric $N \times N$ Matrix, whose elements $[D]_{i j}$ represent the minimum distance or minimum length of the path between the vertices $i$ and $j$ in terms of the number of edges between them.

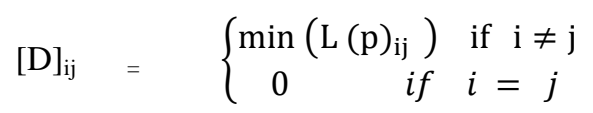

Where $p_{i j}$ is the number of edges separating the vertices $i$ and $j$.

2.6 Minimum Dominating Distance Matrix (S.Vijayalakshmi, M.Raji and G.Jayalalitha ,2018)

Let $\mathrm{D}$ be a minimum dominating set of a graph $\mathrm{G}$. The minimum dominating distance matrix of $\mathrm{G}$ is $\mathrm{N} \times \mathrm{N}$ matrix defined by

$$
\mathrm{A}_{\mathrm{Dd}}(\mathrm{G})=\left(\mathrm{d}_{\mathrm{ij}}\right) \text { where } \mathrm{d}_{\mathrm{ij}}=\left\{\begin{array}{l}
1 \text { if } \mathrm{i}=\mathrm{j} \text { and } \mathrm{v}_{i} \in \mathrm{D} \\
\mathrm{d}\left(v_{i}, v_{j}\right) \quad \text { otherwise }
\end{array} .\right.
$$

\subsection{Molecular Graph Of Anthracene}

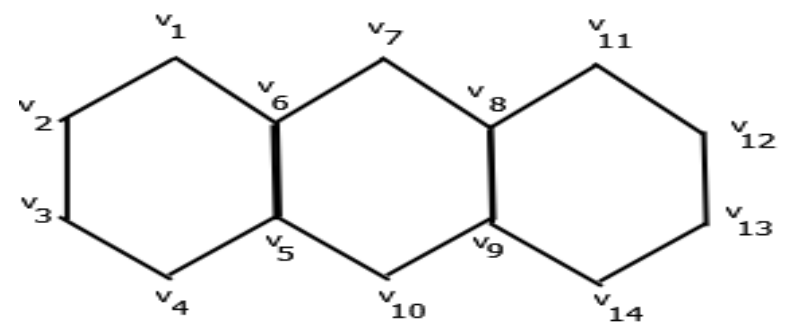

Figure 1.Molecular Graph of Anthracene

2.8 Schultz Polynomial (S. Klavzar, I. Gutman 1996, 1997, H.P. Schultz1989, Mohammad Reza Farahani, Hosoya, 2013):

Schultz Polynomial is defined as

$$
S_{C}(\mathrm{G}, \mathrm{X})=\sum_{u, v \in V(G)}(d u+d v) x^{d(u, v)}
$$

Where $\mathrm{du}, \mathrm{dv}$ are degree of vertex $\mathrm{u}$ and $\mathrm{v}$ for the vertex set $\mathrm{V}(\mathrm{G})$ respectively.

\subsection{Schultz Index}

Harry P. Schultz introduced Schultz index in 1989 and Schultz Index is defined by

$S_{C} \mathrm{G}=\sum_{u, v \in V(G)}(d u+d v) d(u, v)$

Where $d u, d v$ are degree of vertex $u$ and $v$ for the vertex set $V(G)$ respectively.

\subsection{Modified Schultz Polynomial}

Modified Schultz Polynomial is defined as

$\boldsymbol{S}^{*}{ }_{\boldsymbol{C}}(\mathrm{G}, \mathrm{X})=\sum_{u, v \in V(G)}(d u . d v) x^{d(u, v)}$

Where $\mathrm{du}, \mathrm{dv}$ are degree of vertex $\mathrm{u}$ and $\mathrm{v}$ for the vertex set $\mathrm{V}(\mathrm{G})$ respectively.

\subsection{Modified Schultz Index}




\section{Available online at www.ijrat.org}

S. Klavžar and I. Gutman in 1997 introduced the Modified Schultz Index of G is defined as:

$$
\boldsymbol{S}_{C_{C} \mathrm{G}}^{*}=\sum_{u, v \in V(G)}(d u . d v) d(u, v)
$$

Where $d u, d v$ are degree of vertex $u$ and $v$ for the vertex set $V(G)$ respectively.

\section{MAIN RESULTS}

In Section 3.1explains Based on the Molecular Graph of Anthracene(2.7),it finds the degree of each vertex for the Molecular Graph of Anthracene (Table 1), and also in Section 3.2. Based on definition of Minimum Dominating Distance Matrix (2.6), it finds Minimum Dominating Distance Matrix of Anthracene, and also in Section 3.3. Based on the distance

$\mathrm{d}(\mathrm{u}, \mathrm{v}) \quad$,

$\sum_{u, v \in V(G)}(d u+d v), \quad \sum_{u, v \in V(G)}(d u . d v)$ for every vertex $u$ and $v$ for the vertex set $V(G)$ respectively of the molecular graph of Anthracene and in Section 3.4. Based on Minimum Dominating Distance Matrix of Anthracene, it finds Schultz Polynomial, Modified Schultz Polynomial and their Indices of the molecular graph of Anthracene.

In the molecular graph of Anthracene, from figure

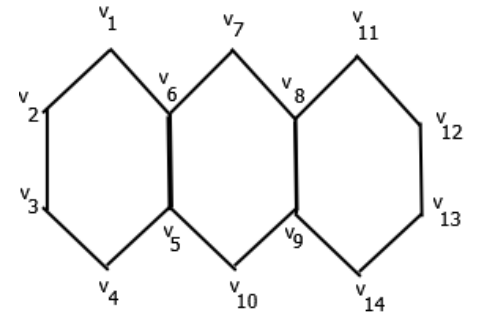

Here $\mathrm{V}=\left\{\mathrm{v}_{1}, \mathrm{v}_{2}, \mathrm{v}_{3}, \mathrm{v}_{4}, \mathrm{v}_{5}, \mathrm{v}_{6}, \mathrm{v}_{7}, \mathrm{v}_{8}, \mathrm{v}_{9}, \mathrm{v}_{10}, \mathrm{v}_{11}, \mathrm{v}_{12}, \mathrm{v}_{13}, \mathrm{v}_{14}\right\}$

Dominating set $\mathrm{D}=\left\{\mathrm{v}_{3}, \mathrm{v}_{6}, \mathrm{v}_{9}, \mathrm{v}_{12}\right\}$ and $\mathrm{V}-\mathrm{D}=\left\{\mathrm{v}_{1}, \mathrm{v}_{2}, \mathrm{v}_{4}, \mathrm{v}_{5}, \mathrm{v}_{7}, \mathrm{v}_{8},, \mathrm{v}_{10}, \mathrm{v}_{11}, \mathrm{v}_{13}, \mathrm{v}_{14}\right\}$.

3.1 Table 1 gives the degree of each vertex for the Molecular Graph of Anthracene.

Table 1.Degree of Molecular Graph

\begin{tabular}{|c|c|c|c|c|c|c|c|c|c|c|c|c|c|c|}
\hline Vertex & $\mathrm{V}_{1}$ & $\mathrm{~V}_{2}$ & $\mathrm{~V}_{3}$ & $\mathrm{~V}_{4}$ & $\mathrm{~V}_{5}$ & $\mathrm{~V}_{6}$ & $\mathrm{~V}_{7}$ & $\mathrm{~V}_{8}$ & $\mathrm{~V}_{9}$ & $\mathrm{~V}_{10}$ & $\mathrm{~V}_{11}$ & $\mathrm{~V}_{12}$ & $\mathrm{~V}_{13}$ & $\mathrm{~V}_{14}$ \\
\hline Degree & 2 & 2 & 2 & 2 & 3 & 3 & 2 & 3 & 3 & 2 & 2 & 2 & 2 & 2 \\
\hline
\end{tabular}


International Journal of Research in Advent Technology, Vol.7, No.1, January 2019

E-ISSN: 2321-9637

Available online at www.ijrat.org

3.2 Minimum Dominating Distance Matrix of Anthracene.

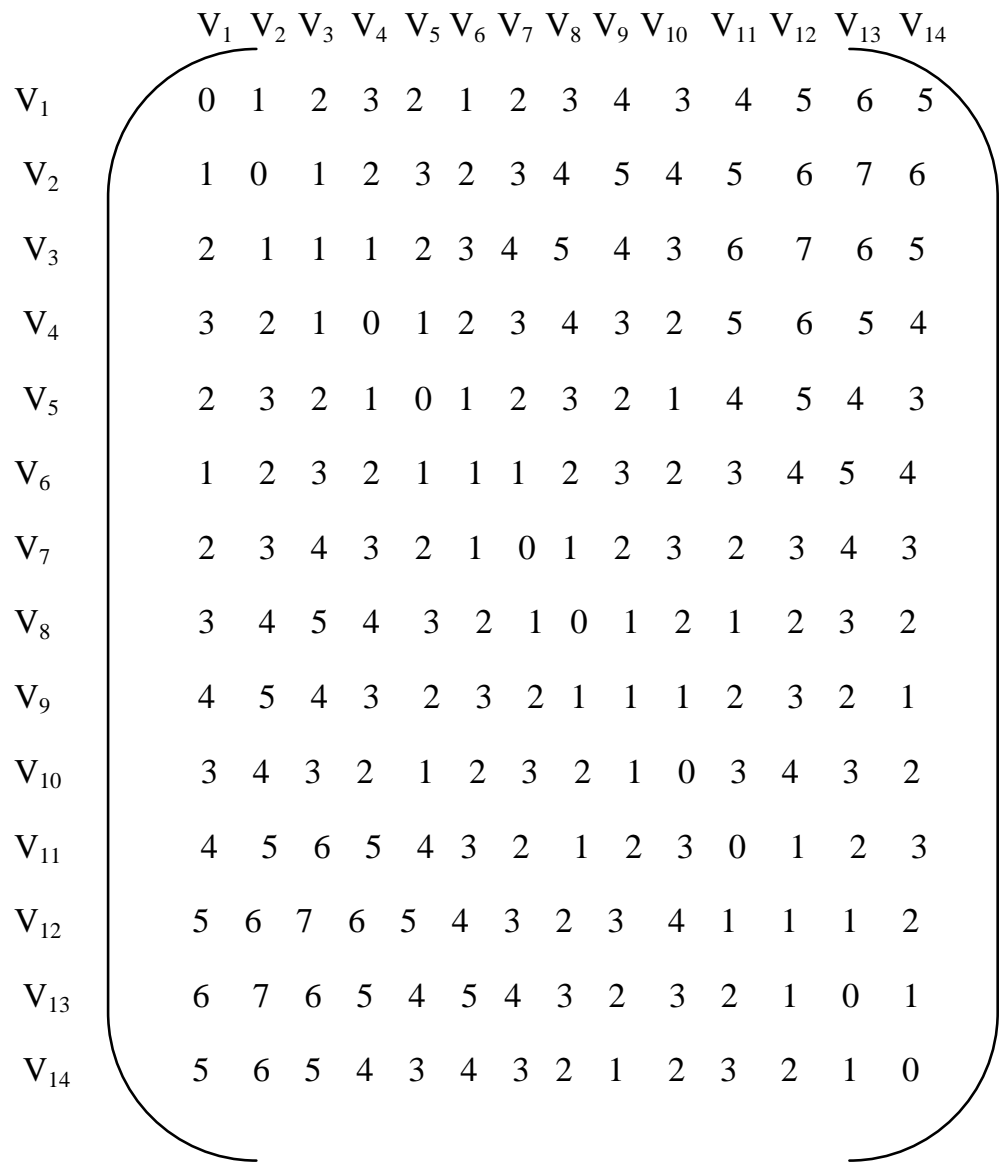

3.3 Table 2 gives the distance $d(u, v), \sum_{u, v \in V(G)}(d u+d v), \sum_{u, v \in V(G)}(d u . d v)$

For every vertex $\mathrm{u}$ and $\mathrm{v}$ for the vertex set $V(G)$ respectively of the Molecular Graph of Anthracene,

TABLE 2.Distance of Molecular Graph

\begin{tabular}{|c|c|c|c|c|c|c|c|}
\hline The distance d(u, v) & 1 & 2 & 3 & 4 & 5 & 6 & 7 \\
\hline$\sum_{u, v \in V(G)}(\boldsymbol{d u}+\boldsymbol{d} \boldsymbol{v})$ & 172 & 208 & 192 & 128 & 88 & 48 & 16 \\
\hline$\sum_{u, v \in V(\boldsymbol{G})}(\boldsymbol{d u} \cdot \boldsymbol{d} \boldsymbol{v})$ & 206 & 244 & 220 & 144 & 96 & 48 & 16 \\
\hline
\end{tabular}

3.4 Schultz Polynomial, Modified Schultz Polynomial and their Indices of the Molecular Graph of Anthracene

From Equation (1), Schultz Polynomial of Anthracene of Minimum Dominating Distance Matrix

$$
S_{C}(G, X)=86 X+104 X^{2}+96 X^{3}+64 X^{4}+44 X^{5}+24 X^{6}+8 X^{7} .
$$

From Equation (3), Schultz Index of Anthracene of Minimum Dominating Distance Matrix 
International Journal of Research in Advent Technology, Vol.7, No.1, January 2019

E-ISSN: 2321-9637

Available online at www.ijrat.org

$S_{C} G=86+104(2)+96(3)+64(4)+44(5)+24(6)+8(7)=1266$.

From Equation (2), Modified Schultz Polynomial of Anthracene of Minimum Dominating Distance Matrix

$$
S_{C}^{*}(G, X)=103 X+122 X^{2}+110 X^{3}+72 X^{4}+48 X^{5}+24 X^{6}+8 X^{7} \text {. }
$$

From Equation (4), Modified Schultz Index of Anthracene of Minimum Dominating Distance Matrix

$$
S_{C}^{*} \mathrm{G}=103+122(2)+110(3)+72(4)+48(5)+24(6)+8(7)=1405 .
$$

\section{CONCLUSION}

This Paper obtained Minimum Dominating Distance Matrix Of Anthracene, Schultz Polynomial, Modified Schultz Polynomial and their Indices of the Molecular graph of Anthracene.

\section{REFERENCES}

[1] Arumugam.S.,Invitation to Graph theory, Scitech Publications (India) Pvt Ltd, 2014, ISBN 978-81-8371-543-0.

[2] S.Vijayalakshmi, M.Raji and G.Jayalalitha, Degree based in Molecular Graph of Organic compounds on Domination, Jour. Of Adv Research in Dynamical \& Control systems, Vol.10, 06-special issue,pp.962-966,(2018).

[3] S. Klavzar, I. Gutman, A Comparison of the Schultz Topological Index with the Wiener Index, J. Chem. Inf. Comput. Sci., 36, 10011003. (1996).

[4] S. Klavžar and I. Gutman. Wiener number of vertex-weighted graphs and a chemical application. Disc. Appl. Math. 80 , 73-81,(1997)

[5] NisreenBukhary,Domination in Benzenoids. Virginia Commonwealth University, VCU Scholars Compass. ,(2010).

[6] Qian-Nan Hu, Yi-Zeng Liang and Kai-Tai Fang, The Matrix Expression. Topological Index and Atomic Attribute of Molecular Topological Structure, Journal of Data Science1.361-369, (2003).

[7] H.P. Schultz, Topological organic chemistry 1. Graph theory and topologicalindices of alkanes. J. Chem. Inf. Comput. Sci. 29, 227-228,(1989).

[8] Mohammad Reza Farahani, Hosoya, Schultz, Modified Schultz Polynomials and Their Topological Indices of Benzene Molecules: First Members of Polycyclic Aromatic Hydrocarbons (PAHs) International Journal of Theoretical Chemistry Vol. 1, No. 2 , PP: 09 - 16, (October 2013).

[9] S. Priyanga, M. Raji and G. Jayalalitha,Domination and Fractional Domination in Molecular Graph of Anthracene,International Journal of Pure and
Applied Mathematics Volume 119 No. 16 49634970,(2018).

[10] Damir vukicevic, Nenad Trinajstic, Wiener Indices of Benzenoid Graphs, Bulletin of the chemists and technologists of Macedonia, vol. 23, No:2, PP:113-129 (2004).

[11] Teresa W.Haynes,Stephen T. Hedetniemi,Peter J. Slater, Fundamentals of Domination in Graphs, Marchel Dekker,ISBN 0-8247-00333,(1998). 